\title{
Computerised axial tomography in patients with severe migraine: a preliminary report
}

\author{
G. D. HU NGERFOR D ${ }^{1}, G$. H. d u B O U L A Y ${ }^{2}$, \\ A N D K . J . Z I L K H A \\ The National Hospital for Nervous Diseases, Queen Square, London
}

SYNOPSIS Patients suffering from severe migraine, usually for many years, have been examined by the EMI scanner between attacks. Judged by criteria validated originally by comparison with pneumoencephalography, about half of the patients showed evidence of cerebral atrophy. Perhaps of more significance than generalised atrophy was the frequency of areas of focal atrophy and of evidence of infarction.

Although migraine is a common disease afflicting between $5 \%$ and $10 \%$ of the population (Friedman, 1971), little is known about either the underlying pathological changes which are present during an attack or the structural changes, if any, which may occur in the brain after one or many episodes. This difficulty in obtaining information arises from the fact that migraine is rarely a fatal illness, and such information as we have is derived from studies of those vessels which are visible in the retina, from the very small number of postmortem studies which have been reported in patients dying from migraine and its complications, and from a few angiographic and cerebral blood flow studies.

Computerised axial tomography (CAT) (EMI scanning) offers a new opportunity for the visualisation of pathological changes in the brain occurring in the course of non-fatal illness.

Symonds (1951) suggested that slight, but cumulative structural damage may result from repeated attacks of migraine. We have therefore analysed the results of CAT in a group of patients suffering from severe migraine in an attempt to determine whether structural damage does occur.

${ }^{1}$ Present address: Department of Radiology, Medical University of South Carolina, Charleston, South Carolina 29401, USA.

${ }^{2}$ Address for correspondence: Professor G. H. du Boulay, The National Hospital, Queen Square, London WC1N 3BG.

(Accepted 10 June 1976.)

\section{METHODS}

Fifty-three patients (36 females, 17 males) wer8 studied. Their average age was 42.7 years. Th basic criteria for inclusion in this group were that the migraine had either been exceptionally severe or had been accompanied by serious clinicas complications. Many cases were of very lon standing. The average duration of the disordef since onset was 20 years. Patients with migrainous neuralgia were specifically excluded because of its uncertain relationship to classical migraine. No patient was examined in the height of an attack. The machine used was an EMI scanner with a $160 \times 160$ matrix.

\section{RESULTS}

The CAT scans of 28 patients were normal (Table). The mean duration of the disorder since onset in these patients (18 years) was similar to

T A B L E

RESULT OF SCANS IN 28 PATIENTS

\begin{tabular}{lccccc}
\hline & \multicolumn{5}{c}{ EMI scans } \\
\cline { 2 - 5 } & Normal & \multicolumn{2}{c}{ Atrophy } & Infarct & Other \\
\cline { 2 - 4 } Migraine & & General & Focal & & \\
\hline Severe & 22 & 4 & 7 & 3 & 4 \\
Hemiplegic & 6 & 2 & 1 & 3 & 1 \\
Total & 28 & 6 & 8 & 6 & 5 \\
\hline
\end{tabular}


that for the group as a whole, but the mean age of the normal patients at the time of study was five years less than the mean age for the whole group. It might be expected that the patients with normal CAT scans would have less severe migraine than those patients whose CAT scans were abnormal, but on analysis this does not appear to be so.

It is notable that the group with normal CAT scans included six patients who had suffered from hemiplegic migraine. One such patient had had left hemiparesis for six days, another had had approximately seven episodes of alternating hemiparesis in five years, with weakness persisting for periods ranging from two weeks to three months, and a third patient had had many transient episodes of hemiparesis, sometimes more than one per week, over the preceding year.

Thus, in this series at least, there was at first sight little correlation between the CAT scan appearance and the duration of disease or severity of episodes, but it should be remembered that all patients included in this study suffered from severe or very severe migraine, and we are not yet in a position to compare migraine sufferers with age-matched controls. The findings that nearly half the patients had abnormal CAT scans must be worrying.

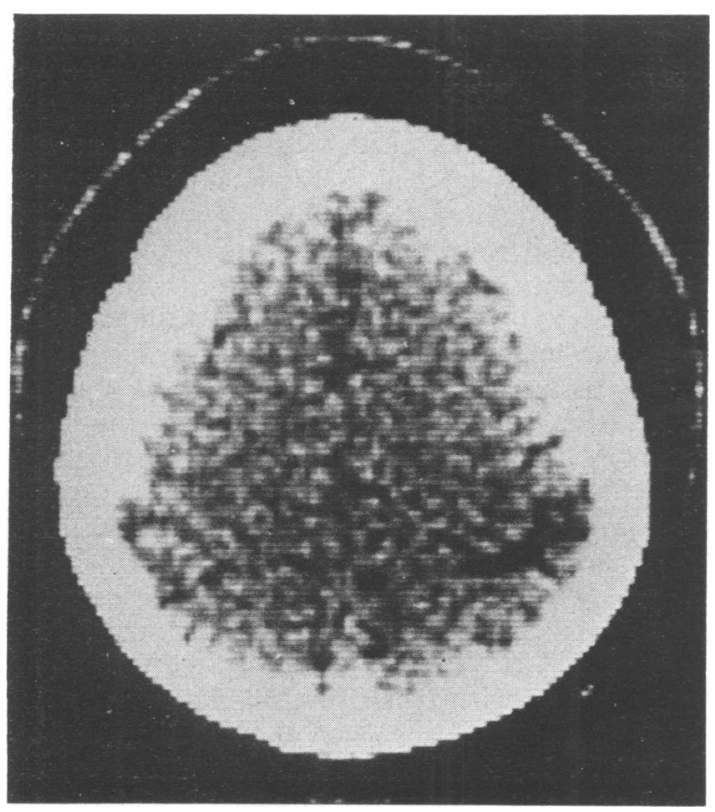

FIG. 1 Focal atrophy in the right parietal region in a 27 year old man.
In a further attempt to find a correlation between clinical findings and CAT scan appearance, the relevance of permanent neurological sequelae of migraine was explored. Here a clear correlation was evident. Thirteen of our patients had permanent neurological sequelae and, of these, 11 had abnormal CAT scans. There was a tendency for the severity of the clinical sequelae to correlate with the degree of abnormality on the CAT scan. Thus the two patients who had normal CAT scans were one who had a central scotoma in the left eye and one who had mild bilateral visual impairment with minimal clumsiness of the right hand. By contrast there were five patients with residual hemiparesis or hemiplegia, all of whom showed abnormalities which, in some cases, were striking. The difficulty in this group with permanent neurological sequelae is to exclude other vascular causes for the pathological changes.

There were two principal types of abnormal findings on the CAT scans of patients with and without permanent neurological sequelae as well as a heterogeneous collection of unrelated abnormalities. These two principal abnormalities were cerebral atrophy and cerebral infarction.

\section{ATROPHY}

Fourteen patients showed unequivocal evidence of cerebral atrophy (enlarged ventricles and/or cortical subarachnoid spaces) judged by evidence validated by Gawler et al. (1976) in comparison with pneumoencephalograms. Within this group was a man aged 27 (Fig. 1) and another aged 29 years.

\section{INFARCTION}

The diagnosis of an infarct is made when an area of brain tissue, often wedge-shaped and more or less representing a vascular or watershed territory, becomes translucent to $\mathrm{x}$-rays and is associated with an appropriate clinical picture. The translucency may evolve over 24-48 hours, sometimes with some associated swelling. Thereafter it diminishes in extent at the same time as it becomes more translucent. Ventricles and subarachnoid spaces begin to show evidence of displacement towards the lesion. In about three weeks the infarct is mature, a well-defined, intracerebral, low-density region, usually with evidence of localised brain atrophy. Such lesions were seen in six patients. As with atrophy, there is some difficulty in determining the degree to which migraine could be convicted of causing the change, but in two or perhaps three of the six 
the relationship of the clinical story and the angiographic and CAT scan appearances strongly suggests that migraine was causative. The first patient, in whom the association was very strong, was a 30 year old man with a 10 year history of migraine which previously had not been associated with visual disturbance. After one episode he developed a permanent right upper quadrantanopia, and at angiography was found to have occlusion of the left posterior cerebral artery. His CAT scan showed a left occipital infarct. The second patient was similar to the first and showed an infarct of the left posterior temporal region (Fig. 2). The third patient, one in whom the possibility of other small vessel disease also had to be considered, was a woman of 54 years who had had migraine on and off for 43 years, her attacks being associated with left hemiplegia, dysphasia, hemianopia, and loss of consciousness. A right carotid angiogram in 1971 showed vessels of irregular calibre with some narrowed segments. Her CAT scan showed extensive, bilateral areas of translucency which we have interpreted as infarction (Fig. 3).

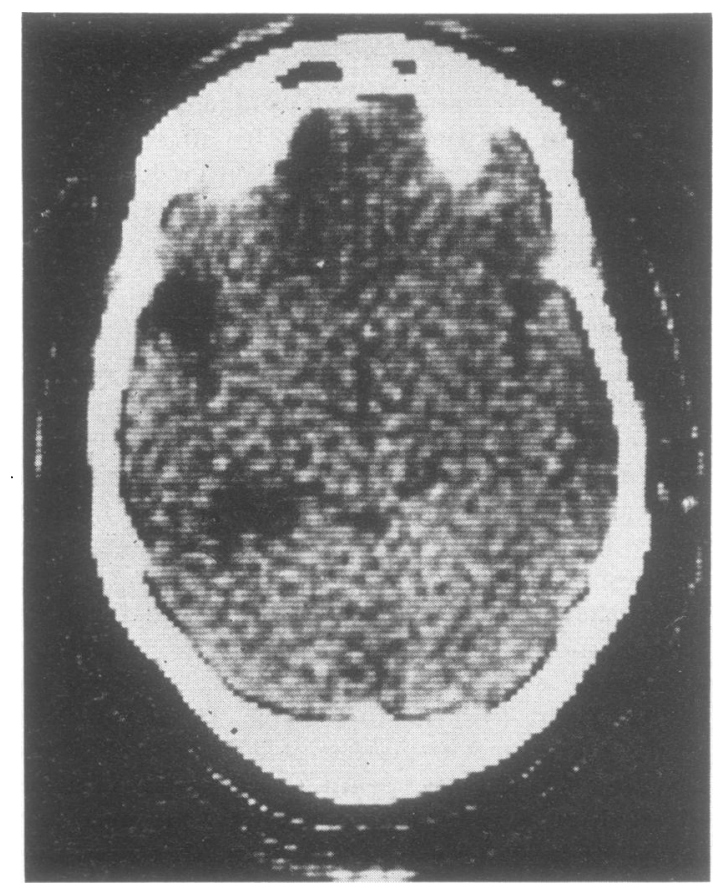

FIG. 2 Posterior temporal infarct in a 42 year old woman.

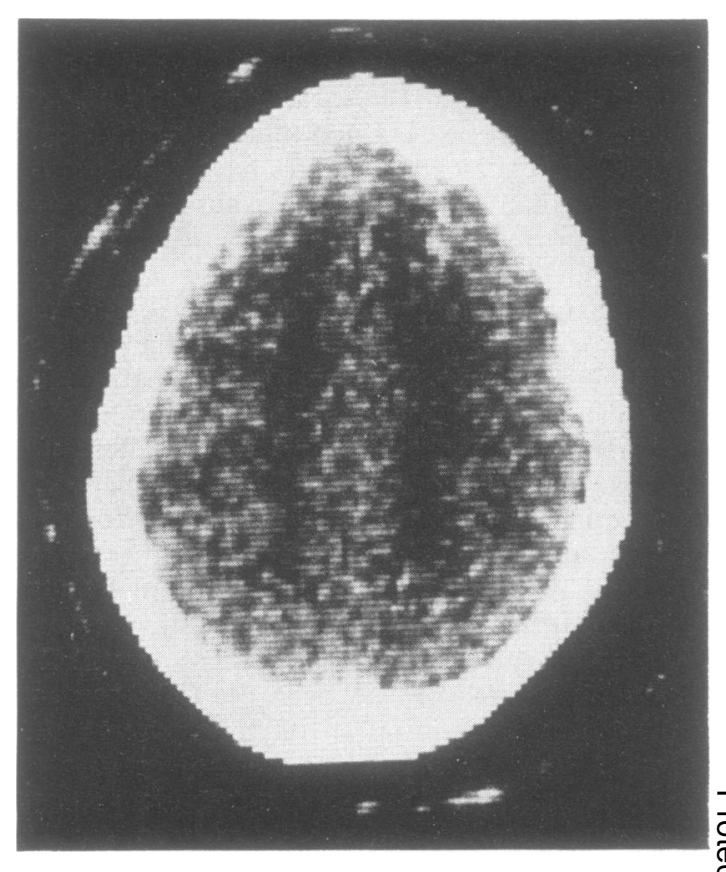

FIG. 3 Extensive bilateral areas of translucency $\frac{0}{\mathrm{D}} \mathrm{O}$ in a 50 year old woman.

The remaining three patients had infarcts in association with migraine, but migraine was not necessarily the sole or, even, a causal factor. One of these, a woman aged 44 years, was found to have severe bilateral intracranial carotid stenoses: she had suffered a double hemiplegia. The CAT scan showed severe atrophy with bilateral areas of infarction.

Another, a 49 year old female, had suffered with migraine for many years. She had awoken with a severe pain which was unlike her usual migraine and was found to have a permanent hemianopia. The CAT scan showed a left parieto-occipital infarct. She was thought to have had a vascular occlusion, but because of the unusual nature of the pain at the time of its onset it was difficult to be sure whether or not migraine was the cause.

The last patient who suffered a definite infarct was a 43 year old male who, on a carotid angiogram, showed marked narrowing of the left internal carotid artery one inch $(2.5 \mathrm{~cm})$ above its origin. He had had migraine for 36 years, and 10 days before his angiogram had had left neck pain which had developed into migraine, accompanied by dysphasia, which lasted for 12 hours. 
Four days after this he developed sudden right hemiplegia and aphasia.

\section{OEDEMA}

One other patient needs to be mentioned here, a 57 year old man with a 47 years history of vertebrobasilar migraine who had developed signs which suggested a left cerebral infarct. His CAT scan showed curious bilateral areas of decreased density in the cerebral hemispheres which may have represented oedema.

\section{HEMIPLEGIC MIGRAINE}

There were 13 patients with hemiplegic migraine, defined as loss of power in one or both limbs on the affected side. Six of these had normal CAT scans, two had generalised atrophy, and three had infarcts (one of these was the previously described patient with the severe narrowing of the internal carotid artery). Eight of the patients had permanent neurological deficits, and of these two had normal CAT scans and two showed infarction. The duration of illness for the patients with hemiplegic migraine was slightly greater than that for the group as a whole, but there was one 12 year old patient who had begun to have attacks of alternating hemiplegia at the age of 3 years. Despite slight residual left-sided weakness after at least three episodes of hemiplegia, which had persisted for periods of up to three months, this patient's CAT scan was normal. His migraine was of the vertebrobasilar type.

\section{ERGOT}

The relationship of atrophy and infarction to treatment is of interest. These patients had had a wide variety of medication. One can say, however, that of the 22 cases with normal CAT scans in whom the history of medication was known, 12 had had an ergot preparation. Likewise, five out of eight cases with atrophy had had ergot. Of those patients with infarcts the drug history was known only in two cases, neither of whom had been given ergot.

\section{DISCUSSION}

It has been suggested previously (Symonds, 1951) that slight, but cumulative structural damage in the brain occurs as a result of repeated attacks of migraine, and Bradshaw and Parsons (1965) have emphasised that, although residual sequelae are rare, the fact that they are sometimes seen suggests that infarction has occurred. Remarkably few necropsy studies are available. Guest and Woolf (1964) reported the case of a 28 year old man who died after a migraine episode. He had brain stem ischaemia and infarction of left frontal gyri which, in the absence of visible arterial occlusion, were thought to be due to arterial spasm. Peters (1934) reported a case of cerebral haemorrhage in a patient with headaches which were typical of migraine, but other features of the history and findings are most unusual and the diagnosis of migraine must therefore be questioned. Whitty (1953) reported a case of Oppenheim's in 1890 , which at necropsy showed extensive thrombosis of the left middle cerebral artery. Buckle et al. (1964) described a patient who died from cerebral infarction after an episode which might have been migraine. The angiogram showed severe bilateral spasm. No other cause for death was found.

Cerebral arteriography has been performed in many patients with migraine (Bradshaw and Parsons, 1965; Blend and Bull, 1967; O'Connor, 1973) and, apart from those cases which were found to have arteriovenous malformations, the great majority were normal. Dukes and Vieth (1964), however, found a progressive decrease of filling of the internal carotid system during the prodromal phase, with a normal angiogram during the succeeding headache, and these findings are in accord with those of cerebral blood flow measurements (Skinhøj, 1971).

Bradshaw and Parsons (1965) found reports of eight patients who had had pneumoencephalography; six were normal and two had changes which might have indicated slight cerebral atrophy. There have been several reports of retinal artery occlusion, but changes in the retinal arteries are not necessarily the same as those in cerebral arteries. Thus, confirmatory evidence of the clinical suspicion of atrophy and/or infarction has been lacking.

The frequency with which generalised atrophy has been demonstrated in the present study cannot, of itself, carry much weight when the patients' ages are taken into consideration, and the indications that in this series atrophy is possibly age-related but not related to the severity of the migraine argues against an association. The changes, when generalised, were mild or moderate, rarely severe, and because of the known but poorly documented relationship between brainshrinkage and advancing years no conclusions can be drawn from them. The significance of focal atrophy may be different, however. Focal atrophy is a well-recognised sequel to infarction and on the 
CAT scan can be confused with a mature infarct. One of the signs of a mature infarct is, in fact, focal atrophy.

The evidence of infarction in two young people, in whom hemianopia accompanied and persisted after typical attacks of migraine, reinforces a fact that is already widely accepted-that posterior cerebral branch occlusion may take place during an attack. The interpretation of the angiogram of the third undoubted sufferer from hemiplegic migraine is difficult. Multiple areas of small vessel narrowing have not so far been accepted as part of the condition, but it is tempting to draw a parellel between them and the usual posterior cerebral branch disease. It may also be suggested, though speculatively, in relation to this case as well as others in the series, that transient arterial narrowing is more likely to produce infarction in the presence of other organic vascular disease. This may explain why some cases of hemiplegic stroke in patients with undoubted atheroma commence after an attack of migraine.

Infarction causes focal atrophy. There is a high incidence of focal atrophic changes in the series, with evidence of a relationship between the site affected and the patients' symptoms, and it may be that this characteristic will eventually distinguish these brains from an age-matched series of controls without migraine. An attempt will be made to do this during the next two years.

There are two reassuring features of our small series, in spite of the fact that we have confirmed the association of migraine and cerebral infarction and in spite of the association between hemiplegic migraine and organic changes. These features are (1) that in spite of life-long severe migraine some of the CAT scans were normal, and (2) there is no obvious association between CAT abnormality and medication with ergot.

\section{REFERENCES}

Blend, R., and Bull, J. W. D. (1967). The radiological investigation of migraine. In Background to
Migraine: First Migraine Symposium, 8th-9th November, 1966. Edited by R. Smith. Heinemann: London.

Bradshaw, P., and Parsons, M. (1965). Hemiplegic migraine: a clinical study. Quarterly Journal of Medicine, 34, 65-85.

Buckle, R. M., du Boulay, G., and Smith, B. (1964). Death due to cerebral vasospasm. Journal of Neurology, Neurosurgery, and Psychiatry, 27, 440-444.

Dukes, H. T., and Vieth, R. G. (1964). Cerebral arteriography during migraine prodrome and headache. Neurology (Minneap.), 14, 636-639.

Friedman, A. P. (1971). Clinical Neurology, vol. 2, p. 5. Edited by A. B. Baker and L. H. Baker. Hagerstoun: Maryland.

Gawler, J., du Boulay, G. H., Bull, J. W. D., and Marshall, J. (1976). Computerized tomography (the EMI scanner): A comparison with pneumoencephalography and ventriculography. Journal of Neurology, Neurosurgery, and Psychiatry, 39, 203211.

Guest, I. A., and Woolf, A. L. (1964). Fatal infaretion of brain in migraine. British Medical Journal, 1, 225-226.

O'Connor, P. J. (1973). Strokes in migraine. In Background to Migraine: Fifth Migraine Symposium 14th-15th September, 1972. Edited by J. N. Cumings. Heinemann: London.

Peters, R. (1934). Tödliche Gehirnblutung bei Menstrueller Migräne. Beiträge zur Pathalogischen Anatomie und zur Allgemeinen Pathologie, 93, 209-217.

Skinhøj, E. (1971). Migraine as a state of CBF disregulation. In Brain and Blood Flow. Proceedings of 4th International Symposium on Regulation of Cerebral Blood Flow, London, September 1970. Edited by R. W. Ross Russell. Pitman: London.

Symonds, C. (1951). Migrainous variations. Transactions of the Medical Society of London, 67, 237.

Whitty, C. M. W. (1953). Familial hemiplegic migraine. Journal of Neurology, Neurosurgery, and Psychiatry, 16, 172-177. 